\title{
PLI-TDC: Super Fine Delay-Time Based Physical-Layer Identification with Time-to-Digital Converter for In-Vehicle Networks
}

\author{
Shuji Ohira \\ Nara Institute of Science and Technology, Japan \\ ohira.shuji.ok2@is.naist.jp \\ Ismail Arai \\ Nara Institute of Science and Technology, Japan \\ ismail@itc.naist.jp
}

\author{
Araya Kibrom Desta \\ Nara Institute of Science and Technology, Japan \\ kibrom_desta.araya.js3@is.naist.jp \\ Kazutoshi Fujikawa \\ Nara Institute of Science and Technology, Japan \\ fujikawa@itc.naist.jp
}

\begin{abstract}
Recently, cyberattacks on Controller Area Network (CAN) which is one of the automotive networks are becoming a severe problem. $\mathrm{CAN}$ is a protocol for communicating among Electronic Control Units (ECUs) and it is a de-facto standard of automotive networks. Some security researchers point out several vulnerabilities in CAN such as unable to distinguish spoofing messages due to no authentication and no sender identification. To prevent a malicious message injection, at least we should identify the malicious senders by analyzing live messages. In previous work, a delay-time based method called Divider to identify the sender node has been proposed. However, Divider could not identify ECUs which have similar variations because Divider's measurement clock has coarse time-resolution. In addition, Divider cannot adapt a drift of delay-time caused by the temperature drift at the ambient buses. In this paper, we propose a super fine delay-time based sender identification method with Time-to-Digital Converter (TDC). The proposed method achieves an accuracy rate of $99.67 \%$ in the CAN bus prototype and $97.04 \%$ in a real-vehicle. Besides, in an environment of drifting temperature, the proposed method can achieve a mean accuracy of over $99 \%$.
\end{abstract}

\section{CCS CONCEPTS}

- Security and privacy $\rightarrow$ Embedded systems security; Intrusion detection systems; • Hardware $\rightarrow$ Buses and high-speed links; Networking hardware.

\section{KEYWORDS}

Physical-Layer Identification; Controller Area Networks; Electronic Control Units; Machine Learning; Intrusion Detection Systems

\section{ACM Reference Format:}

Shuji Ohira, Araya Kibrom Desta, Ismail Arai, and Kazutoshi Fujikawa. 2021. PLI-TDC: Super Fine Delay-Time Based Physical-Layer Identification with Time-to-Digital Converter for In-Vehicle Networks. In Proceedings of the

Permission to make digital or hard copies of all or part of this work for personal or classroom use is granted without fee provided that copies are not made or distributed for profit or commercial advantage and that copies bear this notice and the full citation on the first page. Copyrights for components of this work owned by others than the author(s) must be honored. Abstracting with credit is permitted. To copy otherwise, or republish, to post on servers or to redistribute to lists, requires prior specific permission and/or a fee. Request permissions from permissions@acm.org.

ASIA CCS '21, fune 7-11, 2021, Hong Kong, Hong Kong

(C) 2021 Copyright held by the owner/author(s). Publication rights licensed to ACM ACM ISBN 978-1-4503-8287-8/21/06 ..\$15.00

https://doi.org/10.1145/3433210.3437530
2021 ACM Asia Conference on Computer and Communications Security (ASIA CCS '21), June 7-11, 2021, Hong Kong, Hong Kong. ACM, New York, NY, USA, 11 pages. https://doi.org/10.1145/3433210.3437530

\section{INTRODUCTION}

Due to an increase of connected vehicles, cyberattacks on automobiles' networks are becoming a severe problem [16, 19]. These attacks abuse vulnerable Controller Area Network (CAN) [8] which is one of the in-vehicle network protocols that is used to communicate among Electronic Control Units (ECUs) and has been de-facto standard. Recently, Tencent Keen Security Lab successfully compromised Display Control Unit (DCU) from Bluetooth interface [6]. And, they showed that an attacker could send the diagnostic CAN messages that create unexpected motions to the vehicle. Therefore, cybersecurity countermeasures for automobiles are urgently required.

Encryption and authentication for CAN have been proposed to prevent spoofing attacks from a compromised ECU. However, since CAN has only a short data field of 8 bytes and limited bandwidth, adding a Message Authentication Code (MAC) is not practical. Besides, these approaches required additional hardware and revising some source codes in ECUs to encrypt CAN messages. Thus, it is difficult that these approaches are adapted to CAN or existing vehicles in terms of applicability and implementation cost.

In contrast, some Intrusion Detection Systems (IDSs) have a good advantage in terms of applicability and implementation cost in automotive security different from encryption and authentication. One such case is, IDSs based on characteristics of digital-level (e.g., frequency [24], entropy [15, 28], ID sequence [14]). The approach can be adapted easily to the CAN bus of modern automobiles. However, these approaches typically have higher false positives for some attack types. For instance, ID sequence-based IDS [14] cannot detect replay attacks, in which an adversary sends messages of the same ID sequence. Besides, the IDS on a CAN using deep learning has been proposed [10, 26]. However, these methods cannot be realized on in-vehicle computers in restricted resources. Hence, we should consider an IDS that can detect various attack types and can be implemented in resource-limited systems.

An IDS based on physical-level features such as delay-time which is a gap time between ideal transition time and actual transition time has been proposed, which is called Divider [22]. Divider identifies sender ECUs based on delay-time measured by a Divider's internal 
clock. Thus, if different ECU's delay-time have similar variations, this approach may not correctly classify legitimate ECUs because the time-resolution of the internal clock is coarse. Therefore, we should focus on enhancing the accuracy of sender identification. In addition, Divider cannot adapt a drift of delay-time caused by the temperature drift. In this research, we propose super fine delaytime based Physical-Layer Identification (PLI) with Time-to-Digital Converter (TDC). The TDC in our method digitizes the delay-time per $154 \mathrm{ps}$. The proposed method realizes temperature-robustness by learning the temperature as one of the features. Also, our proposed method can identify the ECUs with higher accuracy than Divider and at the same sampling count as Divider.

The main contributions of this study can be summarized as follows:

- We propose a PLI using TDC called PLI-TDC. Our method uses new characteristics in the identification of ECUs in CAN. PLI-TDC does not use continuous characteristics such as voltage, but the delay-time to be observed in each rising edge of the CAN message. Hence, PLI-TDC can identify the ECUs with a lower number of sampling than the voltagedomain based method.

- PLI-TDC achieved mean accuracy of $99.67 \%$ and $97.04 \%$ on CAN bus prototype and a real-vehicle network, respectively. And, we showed that this approach achieved a $100 \%$ true positive rate against two attacker models.

- We designed PLI-TDC so that it can be robust against features' drift caused by temperature drift. From our experiment, PLI-TDC can achieve a mean accuracy of over $99 \%$ even if the temperature is drifted.

- PLI-TDC solved the problems of detection based on multiple frames, number of probes, and robustness against feature drift.

\section{IN-VEHICLE NETWORK}

CAN is one of the in-vehicle network protocols that is widely used to communicate among ECUs and has been a de-facto standard. Typical CAN node consists of Micro Controller Unit (MCU), CAN controller, and CAN transceiver. The CAN controller processes various frames according to the CAN protocol. The CAN transceiver converts the logical level (low and high) and the CAN bus level (dominant and recessive) between the CAN bus and the CAN controller. ISO 11898 gives the High-Speed CAN bus specification. The specifications are given for the maximum baud rate of $1 \mathrm{Mbps}$ and a maximum bus length of $40 \mathrm{~m}$ with up to 30 nodes can be connected. A twisted-pair cable is used to ensure robust noise immunity on the CAN bus. The two wires are called CAN-L and CAN-H respectively. As shown in Fig. 1 (a), the voltage of CAN-H/CAN-L are $3.5 / 1.5 \mathrm{~V}$ and $2.5 / 2.5 \mathrm{~V}$ during dominant and recessive (logical 0 and 1), respectively. Also, a CAN message has a maximum 8 bytes data field whose length is determined by Data Length Code (DLC) in the control field.

As Fig. 1 (b) shows, a CAN data frame does not contain a field that indicates its sender. Hence, a receiver cannot distinguish which ECU transmitted a CAN message. The CAN has several vulnerabilities such as unable to distinguish spoofing messages due to no authentication.

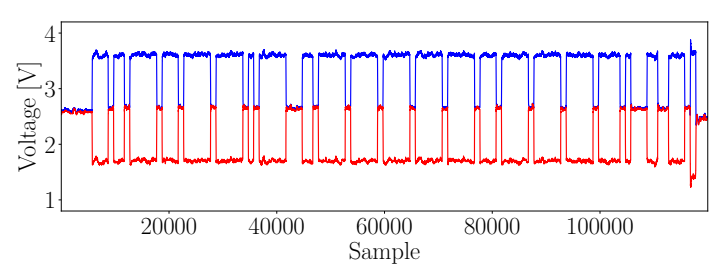

(a) An example of CAN signal.

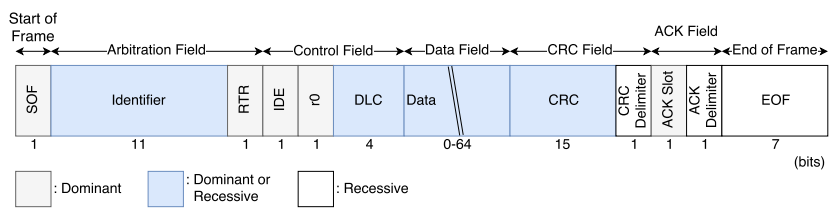

(b) CAN data frame format.

Figure 1: CAN data frame.

\section{RELATED WORKS}

Some authentication mechanisms $[9,20]$ can ensure the authenticity of ECU sending CAN messages. However, these mechanisms required additional hardware and revising some source codes to encrypt CAN messages. Besides, since the authentication mechanisms must manage the key lifecycle based on Public-Key Infrastructure (PKI), it increases the complexity of the automotive system.

PLI can be applied to CAN protocol without these drawbacks. PLI translates some inconsistencies (e.g., hardware and manufacturing) caused by a minute and unique variations to reliable features that can identify ECUs sending CAN messages. Because we do not need to take revising source code and key management into consideration, PLI only requires an additional node to run acquiring fingerprints and classification algorithms.

\subsection{Voltagle-domain based PLI}

Murvay et al. proposed a method for sender identification based on physical voltage features in CAN [17]. And, Choi et al. improved Murvay's method in [5]. They embed a fixed bit string into the extended identifier field of the CAN frame and sample the signal and identify ECUs by using 17 different features. Hence, these methods cannot be implemented on the normal CAN because they require the extended frame format in CAN.

Cho et al. proposed a system for identifying an attacker by using voltage difference among ECUs called Viden [4]. They implemented the system on MCU of a lower sampling rate $(50 \mathrm{kS} / \mathrm{s})$ than CAN bus bit rate. Therefore, Viden requires two or three messages to output a voltage instance and updates the profiles. Thus the receiver cannot help rejecting the first forged message.

Besides, since Viden relies on multiple messages to make detection and identification, Viden has vulnerability against the Hillclimbing-style attack [7], in which an attacker sends gradually malicious messages without being either detected or identified. To be robust against the Hill-climbing-style attack, IDS has to detect the attacks using features acquired in one message $[5,7,11]$.

Scission [11] solved the problem in the sender identification method proposed by Choi et al. [5] which the method could not get 
Table 1: Comparison among time-domain based physicallayer identifications for CAN.

\begin{tabular}{|c|c|c|c|c|}
\hline & हीं & बें & ळे & 㝏 \\
\hline Accuracy [\%] & 99.59 & 100.0 & 87.20 & 99.67 \\
\hline Source & $\begin{array}{c}\text { Bit } \\
\text {-time }\end{array}$ & $\begin{array}{c}\text { Propagation } \\
\text {-delay }\end{array}$ & $\begin{array}{l}\text { Transition } \\
\text {-time }\end{array}$ & $\begin{array}{l}\text { Transition } \\
\text {-time }\end{array}$ \\
\hline One frame & no & yes & yes & yes \\
\hline \# of probe & 1 & 2 & 1 & 1 \\
\hline $\begin{array}{l}\text { Concept drift } \\
\text { robustness }\end{array}$ & yes & no & no & yes \\
\hline
\end{tabular}

significant characteristics such as the overshoot. Scission achieves $99.85 \%$ of identification accuracy which is higher than that of $\mathrm{Vi}^{-}$ den and the method of Choi et al. However, since Scission uses Fourier Transform to calculate the features of the frequency domain, the time complexity of Scission is $\Omega(n \log n)$ which is higher than the time complexity of SIMPLE [7]. Because SIMPLE only uses means of voltage as a feature of ECUs, the time complexity is $\Theta(n)$. Since these sender identification methods use a result of sampling continuous function, the accuracy of identification depends on the sampling rate. In general, as the sampling rate increases, the accuracy of identification is improved. But the amount of data used for the identification increase too. Hence, IDS which is limited in computing resources on the in-vehicle system needs to be able to identify ECUs with few sampling. Therefore, we focus on the sender identification method using other characteristics with few sampling.

\subsection{Time-domain based PLI}

Table 1 shows a comparison among time-domain PLIs.

Bit-time based PLI called BTMonitor [30] has been proposed. This method achieved a mean accuracy of over $99 \%$ based on features extracted from CAN messages ranging from 5 to 50 . Similar to Viden, it cannot help in rejecting the first forged message. In addition, a mean accuracy is $90.04 \%$ if BTMonitor uses the features from one CAN message. Hence, a PLI should achieve a mean accuracy of over $99 \%$ with only one CAN message.

The PLI based on the propagation-delay of wire is caused by wire speed called TIDAL-CAN has been proposed [18]. The method requires at least two probe points for each CAN. The modern invehicle network often divided into multiple parts. Therefore, $2 n$ probe points are required for $n$ CANs in this method. It ruins the simplicity of CAN bus due to the requirement of increasing the probe points. In terms of applicability to real-vehicles, the identification method should have one probe point for each CAN. Further, this method may not be robust against the drift of features caused by temperature change, and so on.

Divider [22] has some advantages in terms of the number of frames used in the detection and the number of probes compared to the other time-domain methods. Divider uses delay-time which is a gap from ideal transition-time to actual transition-time in typical CAN transceiver. If there are some ECUs with similar gaps,

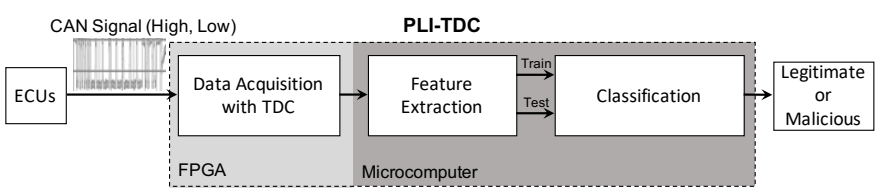

Figure 2: Proposed physical-layer identification.

Divider cannot correctly classify the sender ECU. Also, Divider can distinguish the ECUs with a mean accuracy of $87.20 \%$ in the case of time-resolution $20 \mathrm{~ns}$. Therefore, Divider has a disadvantage in classification accuracy compared to BTMonitor and TIDAL-CAN. It is required that time-resolution in Divider is improved to overcome the problem of classification accuracy. In addition, similar to TIDAL-CAN, Divider is not robust against the drift of features.

In the next section, we propose a fine time-resolution TDC based PLI which has higher accuracy than Divider and overcomes the problems such as needing multi frames and intolerance of temperature change. To solve these problems, we improve Divider by data acquisition using fine time-resolution TDC and adding temperature information as one feature.

\section{TIME-TO-DIGITAL CONVERTER BASED PHYSICAL-LAYER IDENTIFICATION}

PLI-TDC consists of three phases, data acquisition with TDC, feature extraction and classification, as shown in Fig. 2. In the data acquisition phase, the delay-time is acquired as a digital value using TDC. In the feature extraction phase, the delay-time obtained from TDC is converted into statistics such as average, variance, and so on. Finally, it classifies the source ECU of the CAN message to judge whether the ECU is legitimate or malicious. In the following sections, we describe each phase in order.

\subsection{Data acquisition with TDC}

The data acquired by PLI-TDC is the delay-time in the rise- and falltimes of the CAN signal, and its definition and observation method are described below.

4.1.1 Definition of delay-time. The delay-time used in PLI-TDC is the same as Divider's delay-time [22] which is the gap time between actual transition-time and ideal transition time of the signal in CAN transceiver. The Divider's delay-time is caused by the load capacitance of the transistor in CAN transceiver. And the factors of load capacitance include three types of output capacitance at the gate of the transistor, input capacitance of the gate and wiring capacitance. Here, the cause of the delay-time and the equation for calculating the delay-time are described.

A delay model in CAN is showed in Fig. 3. The time variables have the following relation. From the definition, we obtain the following equation.

$$
t_{3}-t_{2}=t_{4}-t_{1}
$$

Here, the time actually measured at the IDS is only $t_{4}$, and $t_{1}, t_{2}, t_{3}$ are unknown. Therefore, we use an approximation. From [22], since $t_{1}$ is regarded as $t_{\text {bit }}$ which is ideal bit time, we obtain the following equation.

$$
t_{3}-t_{2} \approx t_{4}-t_{\mathrm{bit}}
$$




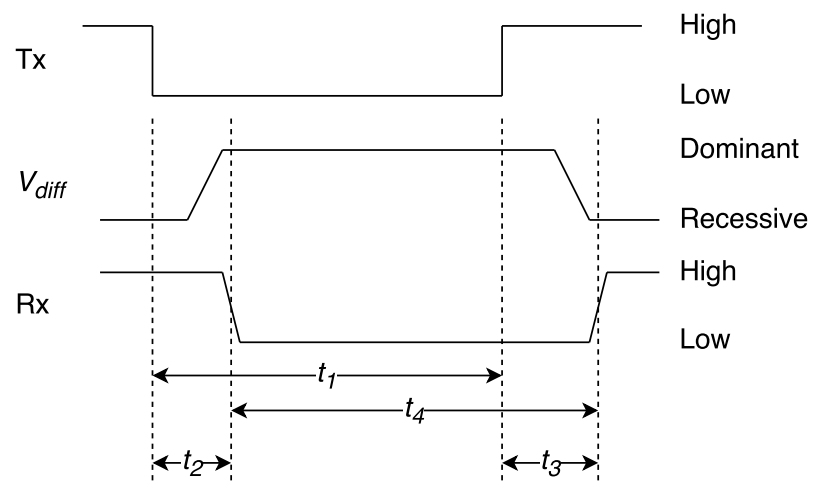

Figure 3: Delay model in CAN.

Also, $t_{\text {bit }}$ is $2000 \mathrm{~ns}$ in CAN (500 kbps). Hence, PLI-TDC acquire delay-times by observing the $t_{4}-t_{\mathrm{bit}}$.

4.1.2 TDC based measurement of delay-time. The TDC is a time digitizer used for physics experiments and time-of-flight (ToF) technique [25]. An IC containing TDC is sold as a product for about $\$ 23.80$ [1]. However, the IC does not satisfy a requirement for PLITDC, because it is necessary to directly control TDC to match the arbitration ID and measured time in PLI-TDC. On the other hand, the methods of implementing TDC at low cost using FieldProgrammable Gate Array (FPGA) have been studied [25, 27]. Also, an arbitration ID and delay-time can be easily matched by implementing TDC in FPGA. Therefore, TDC on FPGA is used to observe the delay-time in CAN with high time-resolution in PLI-TDC.

First, we describe the composition of CMOS based TDC (CMOS TDC) which is one of the typical TDC [2]. Figure 4 (a) shows CMOS TDC circuit. The CMOS TDC is implemented by D-type flip-flops and delay-cells causing a little delay $\tau$ ps. Here, we explain the operation of CMOS TDC. We define $T$ is the measured time. Further, we suppose that there is a signal $\mathrm{Rx}$ (the top of the figure 4 (a)) whose logical value is 0 during the time $T$. This signal $\mathrm{Rx}$ is inputted to the two inputs of CMOS TDC simultaneously. After inputting, as shown in the figure 4 (b), the input signal delayed by the delay-cells by $\tau$ and propagates to the entire CMOS TDC. If the signal Rx rises, the output of the D-type flip-flop to the output $\mathrm{Q}$ and $D_{0} D_{1} D_{2} D_{3}$ are determined. Thus, the output of CMOS TDC $D_{0} D_{1} D_{2} D_{3}=$ $(0,0,0,1)$ is obtained. Here, assuming the delay $\tau=100 \mathrm{ps}$, the signal is propagated from the output $D_{0} D_{1} D_{2} D_{3}=(0,0,0,1)$ to the third delay cell. Therefore, $T=3 \times 100 \mathrm{ps}=300 \mathrm{ps}$. Generally, TDC achieves high time-resolution by the above operation.

Next, we describe the implementation method of FPGA based TDC. Song et al. [25] implemented a delay-line using a multi-bit adder in FPGA. Figure 4 (c) shows the implementation of delayline using a multi-bit adder in Tapped-Delay TDC. The boolean equation of each adder is as follows.

$$
\begin{gathered}
S=A \oplus B \oplus C_{\mathrm{i}} \\
C_{\mathrm{o}}=A B+(A+B) C_{\mathrm{i}}
\end{gathered}
$$

where $A$ and $B$ are the input of the adder, and $C_{\mathrm{i}}$ (carry-in bit) is the input carried from the previous adder, $C_{\mathrm{o}}$ (carry-out bit) is the output carrying to the next adder, and $S$ is the result of the addition. Therefore, the delay-line propagate $C_{\mathrm{o}}[0]=1$ when a signal $\mathrm{Hit}$ becomes $\mathrm{Hit}=1$. And then, The output case $S$ of each adder becomes 0 .

We describe that the time-resolution performance of the implemented FPGA based TDC. When a 20 ns pulse was input to the TDC, the signal was transmitted to the 92 delay element. Similarly, when a $40 \mathrm{~ns}$ pulse was measured, the signal was transmitted to 183 delay elements. From this results, a delay with one delay-cell is $\frac{40-20}{183-92} \mathrm{~ns}=219 \mathrm{ps}$. Then, we calculated the root mean square error (RMSE) between the actual value and the true value $20 \mathrm{~ns}$. And we measured the $20 \mathrm{~ns}$ pulse in 50000 times. As a result, it was obtained that the RMSE was 154.011 ps. Therefore, the implemented TDC has a time resolution of 154 ps.

The delay-time experimentally observed by TDC is shown in Fig. 5. Six arbitration IDs are plotted from two ECUs. The arbitration IDs of ECU a is plotted around $50 \mathrm{~ns}$, and the arbitration IDs of ECU b is plotted around $110 \mathrm{~ns}$. Therefore, we confirm that sender identification is possible regardless of the arbitration ID of the CAN message sent from the two ECUs.

4.1.3 Measurement period. As we showed in Fig. 1, length of a CAN data frame is variable and it is set in the DLC field. Therefore, even if the length of the CAN frame is the shortest (DLC $=0)$, it is necessary to reliably be able to measure the section transmitted by the target node. Then, considering CAN frame such as DLC $=0,35$ bits of signal of SOF ( 1 bit), the arbitration field (12 bits), the control field (6 bits) and CRC filed (16 bits) are transmitted by the ACK field. Here, if we include the $\mathrm{CRC}$ delimiter to the measurement period, the rising edge of the ACK slot may be measured. We subtract 1 bit from the 35 bits. Hence, we set the measurement period from SOF to time that passing 34 bits time $(68 \mu \mathrm{s})$. Since the length of 1 frame is not shorter than the CAN frame in case of DLC $=0$, this allows us to reliably measure only the signal of the target node. Also, during the measurement of delay-time, the time capture is performed every rising edge of the $\mathrm{Rx}$ pin.

We describe how to obtain delay-time, $t_{\text {delay }}$ from the measured counter value. As the unit of timer counter value is $0.154 \mathrm{~ns}$, The elapsed time from SOF, $t_{\text {elapsed }}$ (ns) can be calculated as:

$$
t_{\text {elapsed }}=(\text { capture counter value }-\mathrm{SOF} \text { counter value }) \times 0.154
$$

The value of elapsed bits from the SOF at each rising edge can be calculated as follows:

$$
\left\lfloor\frac{t_{\text {elapsed }}+500}{2000}\right\rfloor
$$

where, 500 is added in the numerator to round $t_{\text {elapsed }}$ by $1000 \mathrm{~ns}$, 2000 is the value of $t_{\text {bit }}$ in ns. Also, 500 is offset to obtain the correct elapsed bits. And the ideal value of elapsed bits can be obtained with floor function.

Therefore, the ideal elapsed time from SOF, $t_{\text {ideal }}$ (ns) can be calculated as follows:

$$
t_{\text {ideal }}=\left\lfloor\frac{t_{\text {elapsed }}+500}{2000}\right\rfloor \times 2000
$$

$t_{\text {delay }}(\mathrm{ns})$ we want to calculate is:

$$
t_{\text {delay }}=t_{\text {elapsed }}-t_{\text {ideal }}=t_{\text {elapsed }}-\left\lfloor\frac{t_{\text {elapsed }}+500}{2000}\right\rfloor \times 2000
$$




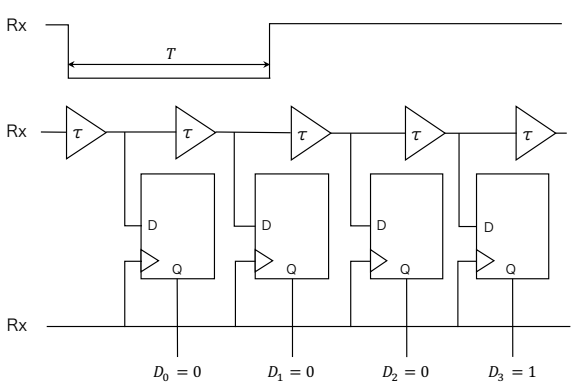

(a) Circuit of CMOS TDC.
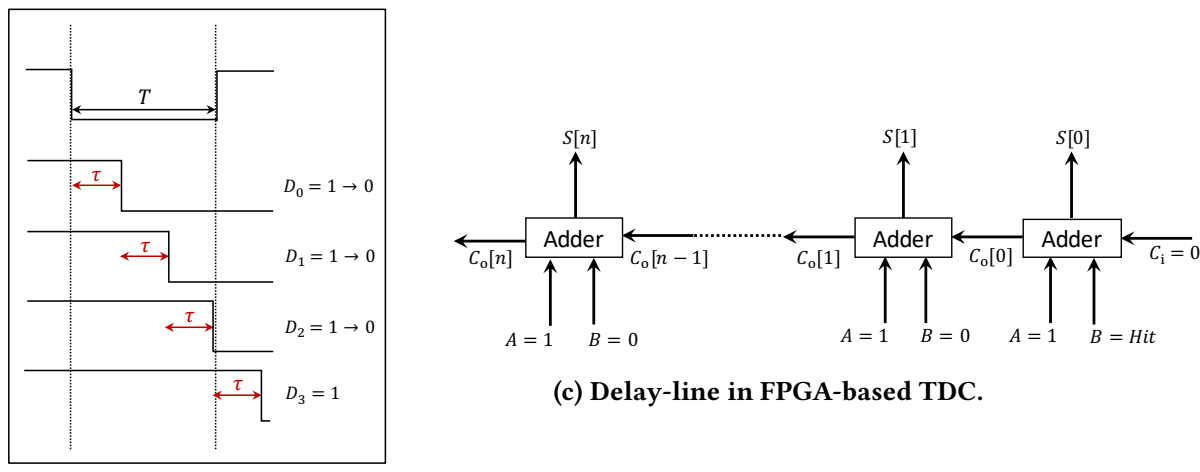

(c) Delay-line in FPGA-based TDC.

(b) Timig-chart of CMOS TDC.

Figure 4: Implementation method and timig-chart of TDC.

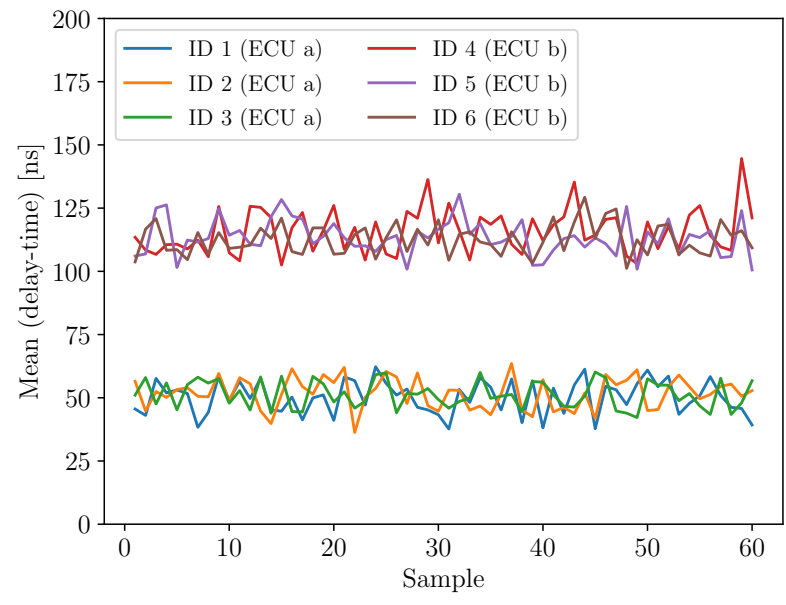

Figure 5: An example of two ECUs' delay-times observed by Time-to-Digital Converter.

Here, we describe the detail of the relation between Equation (2) and (8). Fig. 6 shows an example of the relation in the SOF bit of CAN message. In Fig. 6, the actual signal is observed by IDS and the other ECUs with an unavoidable delay, $t_{\text {delay }}$, which is a gap from $2000 \mathrm{~ns}$ of the ideal signal timing.

We confirm that $t_{\text {delay }}$ equals $t_{4}-t_{\text {bit }}$. First, $t_{\text {elapsed }}$ equals $t_{4}$ because these are times of the dominant signal observed by IDS and the other ECUs. Next, we explain the relation between $t_{\text {bit }}$ and $t_{\text {ideal }} \cdot t_{\text {bit }}$ is an ideal elapsed bit time, and it is $2000 \mathrm{~ns}$ in SOF field of CAN message. In addition, $t_{\text {ideal }}$ indicates the ideal rise time estimated from $t_{\text {elapsed }}$. For example, in case of $t_{\text {elapsed }}=2050 \mathrm{~ns}$, $t_{\text {ideal }}$ is $2000 \mathrm{~ns}$ according to Equation (7). In other words, if $t_{\text {ideal }}$ can be estimated correctly, then $t_{\text {bit }}$ equals $t_{\text {ideal }}$. Therefore, $t_{4}-t_{\mathrm{bit}}$ and $t_{\text {delay }}$ are equal.

\subsection{Feature extraction}

Similar to Scission [11], PLI-TDC selects efficient features from the statistics in Table 2. In order to determine the efficient features, the

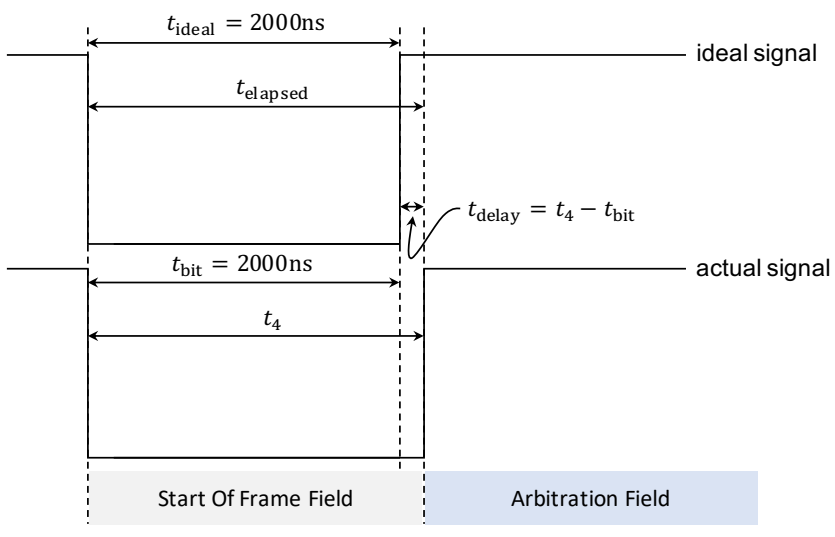

Figure 6: Relation between Equation (2) and (8).

Table 2: A list of statistical features considered in the selection. $x$ is the delay-time in one CAN message, $N$ is the number of measured delay-time in one CAN message.

\begin{tabular}{c|c}
\hline Feature & Description \\
\hline Mean & $\mu=\frac{1}{N} \sum_{i=1}^{N} x(i)$ \\
Standard Deviation (Stdev) & $\sigma=\sqrt{\frac{1}{N} \sum_{i=1}^{N}(x(i)-\mu)^{2}}$ \\
Variance & $\sigma^{2}=\frac{1}{N} \sum_{i=1}^{N}(x(i)-\mu)^{2}$ \\
Skewness & skew $=\frac{1}{N} \sum_{i=1}^{N}\left(\frac{x(i)-\mu}{\sigma}\right)^{3}$ \\
Kurtosis & $k u r t=\frac{1}{N} \sum_{i=1}^{N}\left(\frac{x(i)-\mu}{\sigma}\right)^{4}$ \\
Root Mean Square (RMS) & $r m s=\sqrt{\frac{1}{N} \sum_{i=1}^{N} x(i)^{2}}$ \\
Max & $\max =\max (x(i))_{i=1 \ldots N}$ \\
Min & $\min =\min (x(i))_{i=1 \ldots N}$ \\
Energy & en $=\frac{1}{N} \sum_{i=1}^{N} x(i)^{2}$ \\
\hline
\end{tabular}

features are ranked using Relief-F [13], which is an algorithm that calculates the weight of the features.

Table 3 shows the result of Relief-F in data obtained from a CAN bus prototype and a real-vehicle's bus. In order to reduce the complexity of the model and the time required to calculate the 
Table 3: Ranking of the features calculated by Relief-F [13].

\begin{tabular}{c|cc|cc}
\hline Rank & $\begin{array}{c}\text { CAN bus } \\
\text { prototype }\end{array}$ & Weight & real-vehicle & Weight \\
\hline \hline 1 & Mean & 0.11025 & $\begin{array}{c}\text { Stdev } \\
\text { (fine time) }\end{array}$ & 0.09311 \\
\hline 2 & Min & 0.08773 & Mean & 0.05028 \\
\hline 3 & RMS & 0.05644 & RMS & 0.04833 \\
\hline 4 & Max & 0.04696 & Min & 0.04613 \\
\hline 5 & Kurtosis & 0.03398 & Kurtosis & 0.04090 \\
\hline 6 & $\begin{array}{c}\text { Stdev } \\
\text { (fine time) }\end{array}$ & 0.02949 & Skewness & 0.03694 \\
\hline 7 & Skewness & 0.02307 & Max & 0.02468 \\
\hline 8 & Stdev & 0.01282 & Stdev & 0.01746 \\
\hline 9 & Energy & 0.00878 & Energy & 0.01639 \\
\hline 10 & Variance & 0.00104 & Variance & 0.00723 \\
\hline
\end{tabular}

Table 4: Comparison of machine learning algorithms for PLI-TDC

\begin{tabular}{c|c|c|c|c}
\hline & $\begin{array}{c}\text { Classification } \\
\text { Speed }\end{array}$ & $\begin{array}{c}\text { Training } \\
\text { Speed }\end{array}$ & $\begin{array}{c}\text { Model } \\
\text { Adjustment }\end{array}$ & $\begin{array}{c}\text { Overall } \\
\text { Complexity }\end{array}$ \\
\hline LR & $\checkmark$ & $\times$ & $\checkmark$ & $\checkmark$ \\
Naive Bayes & $\checkmark$ & $\checkmark$ & $\checkmark$ & $\checkmark$ \\
MLP & $\times$ & $\times$ & $\times$ & $\times$ \\
KNN & $\times$ & $\checkmark$ & $\checkmark$ & $\times$ \\
Decision Tree & $\checkmark$ & $\checkmark$ & $\times$ & $\checkmark$ \\
Random Forest & $\checkmark$ & $\checkmark$ & $\times$ & $\checkmark$ \\
SVM (RBF) & $\times$ & $\times$ & $\checkmark$ & $\times$ \\
\hline
\end{tabular}

features, PLI-TDC uses only the features which weight of Relief-F is 0.01 or more in both the CAN bus prototype and the real-vehicle. As a result, eight statistics except energy and variance are selected. In the following, the eight statistics are defined as features.

\subsection{Classification}

Sender identification results in a classification problem. In PLI-TDC, the mean accuracy of various learning algorithms is evaluated. And an algorithm with the highest mean accuracy is used in the classification phase of PLI-TDC.

Here, we describe a comparison of the various learning algorithm. We compare typical learning algorithms composed of function values, distances, trees, and so on. The comparison is summarized in Table 4. The abbreviations express Logistic Regression (LR), Multi-Layer Perceptron (MLP), K-Nearest-Neighbor (KNN), Support Vector Machine (SVM), Radial Basis Function (RBF), respectively. In PLI-TDC, classification speed is essential to identify all messages. LR, Naive Bayes, Decision Tree, and Random Forest meet this requirement. In addition, in in-vehicle networks, some features' drift may be caused by material wear and temperature fluctuations. Therefore, the model should be tolerant of the drift. LR and Naive Bayes may do, but Decision Tree and Random Forest may not. On the other hand, Decision Tree and Random Forest have the advantage of fast training speed in case that the number of trees is few. Therefore, the Decision Tree and Random Forest can adapt the drift by deploying the new model per fixed times.
Therefore, PLI-TDC uses an algorithm with the highest accuracy among these four faster algorithms.

\subsection{Enhancing the concept drift robustness}

In some voltage-based sender identification methods [3, 4, 7, 30], it has been confirmed that the sender's features such as voltage are changed by the drift of temperature. Therefore, we must investigate whether or not the change of delay-time is caused by drift of temperature. We conducted an experimental investigation to determine whether the delay-time has a drift of temperature in Sec. 5.4. As a result, some ECUs had a delay-time that increases monotonically and some ECUs did not show a change in the delay-time. Therefore, PLI-TDC must be robust against the drift of temperature as in the voltage-based source identification method. The approaches to avoid drift of temperature in the voltage-based PLI is as follows.

(1) Correction of features with liner regression [7]

(2) Detection with multi models [30]

(3) Tracking to features' drift [3, 4]

The first method employs linear regression. It cannot be applied to PLI-TDC because the delay-time does not increase linearly with the drift of temperature as described in Sec. 5.4. The second method uses multiple models. It makes the memory usage in a resource-limited system increase compared to one model. Thus, it is ideal to avoid the drift with one model. The third feature tracking method which is used in CIDS [3] and Viden [4] is vulnerable to Hill-climbing-style attack, because they use several CAN message lastly received for learning [7]. Therefore, in the PLI-TDC, the temperature is added as one of the features.

\subsection{Implementation}

In this section, we describe the implementation of PLI-TDC. As mentioned in Sec. 4.1.3, PLI-TDC measures the 34 bits to observe delay-time no matter what length of the data field is received. We show the block diagrams of the implementation of PLI-TDC in Fig. 7 (a). The MCP2551 is a CAN transceiver as the interface between a CAN controller and the physical bus. We also selected an FPGA as a measurement device, because a measurement with software cannot process all messages without missing ones due to the limitation of the ability of microcomputer. We show the prototype of PLI-TDC in Fig. 7 (b). We developed the prototype of the proposed method using FPGA and microcomputer, selected the DE0-CV Cyclone V Board (5CEBA4F23C7) as an FPGA and Raspberry Pi 3 Model B+ as a microcomputer.

Here, we describe the circuits of FPGA in PLI-TDC. The circuits are divided into five operations as follows.

- Coarse Time Sampling Circuit

This circuit measures a period of CAN message in the measurement period of 34 bits with counting per $20 \mathrm{~ns}$.

- TDC Circuit

The TDC circuit measures the period with counting per $154 \mathrm{ps}$. The coarse time sampling and TDC circuit send counting value to a FIFO queue per Rx rising edge of CAN.

- Arbitration ID and DLC Identification Circuit

As its name suggests, we observe and store the arbitration ID and DLC of every message. Similar to the coarse sampling 


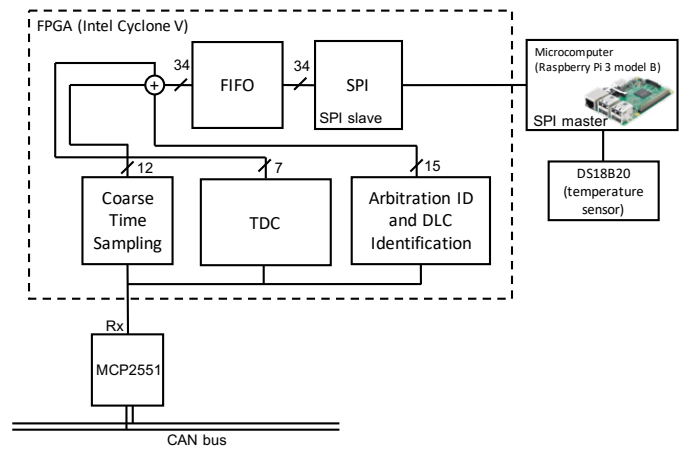

(a) Implementation of PLI-TDC.

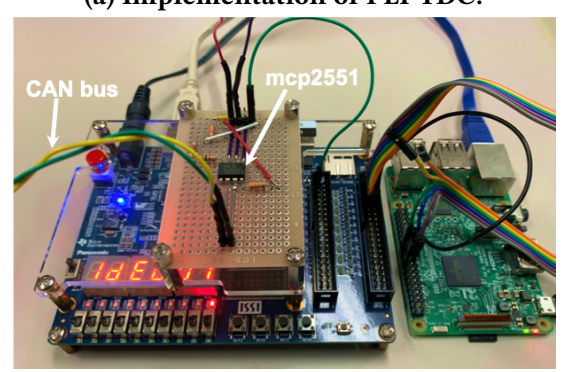

(b) Prototype of PLI-TDC.

Figure 7: Implementation and prototype of PLI-TDC.

circuit, the arbitration ID and DLC sampling circuit sends arbitration ID to FIFO queue per Rx rising edge too.

- FIFO Circuit

In this circuit, we stack the measured data of 34 bits constructed of an arbitration ID of 11 bits, a DLC of 4 bits, and the counter value of coarse / fine time of 19 bits.

- SPI Slave Circuit

We implement the SPI slave module to send measurement data to the Raspberry Pi.

The operation of the measurement is as follows.

(1) Starting the capture of measurement time and arbitration ID, an occurrence at the falling edge of SOF bit.

(2) Send the measurement data (arbitration ID and measurement time) with every rising edge of Rx to a FIFO queue. We calculate the delay-time with equation (8) and record the delay-time after Raspberry Pi receives the measurement data from the FPGA.

(3) After reading 34 bits from SOF, the measurement is ended.

(4) When CAN frame is completely received, the coarse time sampling circuit, TDC circuit, and arbitration ID and DLC identification circuit are waiting SOF bit.

\section{EVALUATION}

In this section, we describe the evaluation of the results. First, we evaluate the accuracy of the identification of ECUs. Second, we experiment on two attacker models and evaluate the attacker detection performance of PLI-TDC. Third, we confirm the robustness of PLI-TDC under different temperatures. Finally, we measure the

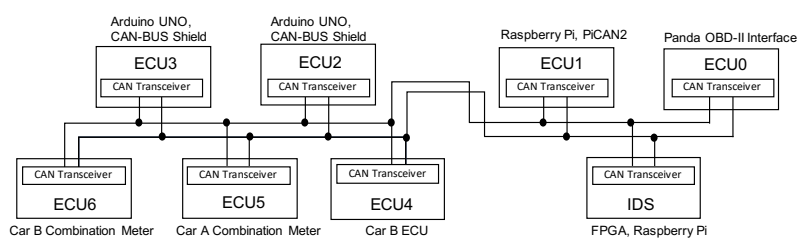

(a) The CAN bus prototype.

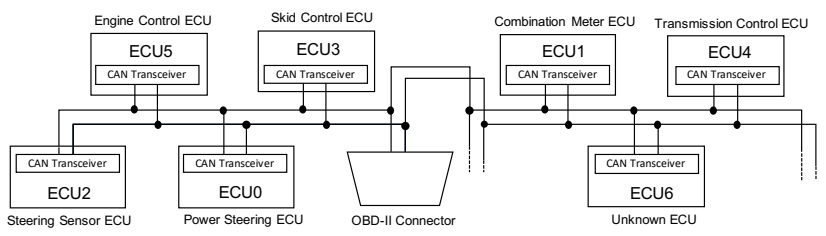

(b) A part of CAN bus in the real-vehicle.

Figure 8: Environments for evaluations.

detection time of PLI-TDC from the feature extraction phase to the classification phase.

\subsection{Environments and attacker models}

In this section, we describe the environments for evaluating PLITDC.

Fig. 8 (a) shows the prototype of the CAN bus topology we implemented in our experiment. We prepare various ECUs to evaluate PLI-TDC. The various ECUs we prepared are described here. As shown in Fig. 8 (a), ECUs 0, 1, 2, and 3 are implemented by microcomputers and dedicated CAN boards. ECU4 is an actual ECU not connected other than CAN, ECUs 5 and 6 are an actual combination meter of each different car model. We cannot control sending CAN messages of ECUs 4, 5, and 6 but these ECUs automatically send some messages periodically, so that PLI-TDC uses the messages to fingerprint ECU.

Fig. 8 (b) shows a part of CAN in real-vehicle which is used to evaluate PLI-TDC. The real-vehicle has multiple CAN buses. One of these CAN buses has a realistic environment in which each ECU has a yaw-rate sensor or an acceleration sensor sends the information to the meter ECU. This CAN bus also has an OBD-II port. In the realvehicle experiment, we have collected the datasets during driving and stopping.

We define two types of attacker models as follows. The first model is based on the hacking of Jeep Cherokee [16]. In actual hacking of Jeep Cherokee, Miller and Valasek exploited a passive or unmonitored ECU's update mechanism to inject their code. Thus, we suppose the type of attacker called unmonitored ECU. By the way, since an ECU has some connectivity interfaces such as Wi-Fi or Bluetooth, some attackers may exploit the attack surfaces such as Wi-Fi or Bluetooth [6]. Therefore, we suppose the attacker model called compromised ECU which is an ECU exploited by the attacker through attack surfaces.

\subsection{Identification of ECUs}

First, we evaluate the mean accuracy of the four algorithms described in Sec. 4.3. We show a result of the evaluation of each 
Table 5: Mean accuracy of each algorithm.

\begin{tabular}{l|c|c}
\hline & \multicolumn{2}{|c}{ Mean accuracy [\%] } \\
\hline Algorithms & CAN bus prototype & real-vehicle \\
\hline LR & 92.86 & 74.83 \\
Naive Bayes & 88.05 & 75.94 \\
Decision Tree & 99.48 & 95.49 \\
Random Forest & $\mathbf{9 9 . 6 7}$ & $\mathbf{9 7 . 0 4}$ \\
\hline
\end{tabular}

Table 6: Confusion matrix for the identification of ECUs of the CAN bus prototype.

\begin{tabular}{|c|c|c|c|c|c|c|c|c|}
\hline & \multicolumn{7}{|c|}{ Predicted label } \\
\hline & & ECU0 & ECU1 & ECU2 & ECU3 & ECU4 & ECU5 & ECU6 \\
\hline \multirow{7}{*}{ 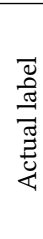 } & ECU0 & 99.04 & 0.06 & 0.00 & 0.00 & 0.00 & 0.89 & 0.00 \\
\hline & ECU1 & 0.06 & 99.78 & 0.06 & 0.00 & 0.00 & 0.11 & 0.00 \\
\hline & ECU2 & 0.00 & 0.00 & 99.94 & 0.06 & 0.00 & 0.00 & 0.00 \\
\hline & ECU3 & 0.00 & 0.00 & 0.06 & 99.94 & 0.00 & 0.00 & 0.00 \\
\hline & ECU4 & 0.00 & 0.00 & 0.00 & 0.00 & 100.00 & 0.00 & 0.00 \\
\hline & ECU5 & 1.29 & 0.00 & 0.11 & 0.00 & 0.00 & 98.60 & 0.00 \\
\hline & ECU6 & 0.00 & 0.00 & 0.00 & 0.00 & 0.06 & 0.00 & 99.95 \\
\hline
\end{tabular}

algorithm in Table 5. Also, we used Random Forest of the number of trees is 50. As the result, we confirmed that Random Forest classifier is the highest accuracy in both CAN bus prototype and real-vehicle. Therefore, we decide that PLI-TDC uses a Random Forest classifier.

Next, we evaluate the mean accuracy with the Random Forest classifier of each ECU in the CAN bus prototype. We have captured 9000 messages from each ECU, dividing the messages into $80 \%$ and $20 \%$ for learning and testing respectively, calculating the eight features from the messages, putting the features into machine learning algorithms. We evaluate the proposed method using K-fold cross-validation in $\mathrm{K}=5$. As a result, the mean accuracy is $99.67 \%$. A confusion matrix in K-fold cross-validation is shown in Table 6. PLITDC can identify correctly with up to $100.00 \%$. While a minimal identification rate is $98.60 \%$ in CAN bus prototype.

We have also evaluated ECU identification accuracy in real vehicle's CAN bus. We have run around the our university $(1.1 \mathrm{~km})$ with $10 \mathrm{~km} / \mathrm{h}$ to $30 \mathrm{~km} / \mathrm{h}$. We have captured 400000 messages from the ECUs. Half of the 400000 messages were observed in stopping and the rest were observed in running. We used the 6010 of the 400000 messages. The number of messages of ECUs $0,1,2,3,4$, and 5 is 1000 messages, respectively. But, the number of ECU6's messages is only 10 messages, because it is non-periodic messages. As with the CAN bus prototype, we divided the CAN messages of the delay-time into learning data and testing data. Hence, we evaluate PLI-TDC using K-fold cross-validation in $\mathrm{K}=5$. From the K-fold cross-validation, PLI-TDC performed well with an average accuracy of $97.04 \%$. A confusion matrix is shown in Table 7. We confirmed that PLI-TDC can identify each ECU correctly with up to $100.00 \%$ while a minimal identification rate is $90.09 \%$.

\subsection{Attacker detection}

In this section, we evaluate the intrusion detection capability of the learned model. To reproduce unmonitored ECU, we attach a
Table 7: Confusion matrix for the identification of ECUs of the real-vehicle.

\begin{tabular}{|c|c|c|c|c|c|c|c|c|}
\hline & \multicolumn{7}{|c|}{ Predicted label } \\
\hline & & ECU0 & ECU1 & ECU2 & ECU3 & ECU4 & ECU5 & ECU6 \\
\hline \multirow{7}{*}{ 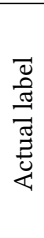 } & ECU0 & 99.50 & 0.00 & 0.00 & 0.50 & 0.00 & 0.00 & 0.00 \\
\hline & ECU1 & 0.00 & 97.97 & 0.00 & 0.00 & 2.03 & 0.00 & 0.00 \\
\hline & ECU2 & 0.00 & 0.00 & 98.50 & 0.00 & 0.00 & 0.15 & 0.00 \\
\hline & ECU3 & 0.00 & 0.00 & 0.00 & 95.31 & 0.00 & 4.69 & 0.00 \\
\hline & ECU4 & 1.99 & 1.99 & 0.00 & 0.00 & 96.02 & 0.00 & 0.00 \\
\hline & ECU5 & 0.47 & 0.00 & 1.41 & 7.55 & 0.00 & 90.09 & 0.00 \\
\hline & ECU6 & 0.00 & 0.00 & 0.00 & 0.00 & 0.00 & 0.00 & 100.00 \\
\hline
\end{tabular}

Table 8: Confusion matrix against sending ID: $x$ from compromised ECU spoofed to ECU3.

\begin{tabular}{r|r|r}
\hline & Predicted: Attack & Predicted: Normal \\
\hline Actual: Attack & 1.0000 & 0.0000 \\
Actual: Normal & 0.0068 & 0.9932 \\
\hline
\end{tabular}

Table 9: Confusion matrix against sending ID: $y$ from compromised ECU spoofed to ECU3.

\begin{tabular}{r|r|r}
\hline & Predicted: Attack & Predicted: Normal \\
\hline Actual: Attack & 1.0000 & 0.0000 \\
Actual: Normal & 0.0570 & 0.9430 \\
\hline
\end{tabular}

new ECU which is ELM327 to the CAN bus prototype. And, we sent an arbitration ID: $x$ assigned in ECU3 from unmonitored ECU spoofed to ECU3. Spoofing attacks were performed for three minutes from unmonitored ECU, and the data during attacks of ECUs were classified by the learned model. The results are shown in Table 8. "Predicted: Attack" is when PLI-TDC classifies messages of ID: $x$ as other than ECU3, "Predicted: Normal" is when PLI-TDC classifies messages of ID: $x$ as ECU3. We confirm the true positive rate against compromised ECU is $100.00 \%$ and the true negative rate is $99.32 \%$.

We evaluate the ability of intrusion detection against compromised ECU. We attached the Arduino UNO (the ECU2 in the prototype of CAN bus) as a compromised ECU in the CAN of the realvehicle. We assume the spoofing attacks of speed information from the compromised ECU. Therefore, the compromised ECU sends ID: $y$ assigned as arbitration ID of speed in the real-vehicle. Spoofing attacks were performed for three minutes from compromised ECU, and the data during sending messages of ECU3 (legitimate ECU of ID: $y$ ) and compromised ECU were classified by the learned model. The results are shown in Table. 9. We confirm the true positive rate against compromised ECU is $100.00 \%$ and the true negative rate is $94.30 \%$.

\subsection{Identification of ECUs under temperature concept drift}

Here, we evaluate PLI-TDC under different temperatures. We used cardboard to cover the CAN bus prototype and increasing the ambient temperature of the CAN bus prototype using a heat-gun. And we corrected data during increasing temperature. We have received 
Table 10: A result of liner regression against temperature drift (CAN bus prototype).

\begin{tabular}{l|cc}
\hline ECU (CAN transceiver) & $R^{2}$ & $M S E$ \\
\hline ECU0 (TJA1040) & 0.0006 & 0.9994 \\
ECU1 (MCP2551) & 0.8544 & 0.1456 \\
ECU2 (MCP2551) & 0.8242 & 0.1758 \\
ECU3 (MCP2551) & 0.6947 & 0.3053 \\
ECU4 (TJA1040) & 0.0948 & 0.9052 \\
ECU5 (SE706) & 0.0706 & 0.9294 \\
ECU6 (TJA1042) & 0.0102 & 0.9898 \\
\hline
\end{tabular}

100000 messages from seven ECUs in the CAN bus prototype. We use the messages to calculate R-squared $\left(R^{2}\right)$ in case that $X$ is temperature and $Y$ is delay-time. The average $R^{2}$ and mean square error (MSE) are given in Table 10. It shows the $R^{2}$ and MSE using the data from $30{ }^{\circ} \mathrm{C}$ to $45^{\circ} \mathrm{C}$. The $R^{2}$ varies depending on the type of CAN transceiver. Therefore, a learned model must respond to feature drift caused by drift of temperature for ECUs 1, 2, and 3.

Furthermore, we validated a new robust model for which temperature is added as one of the features. First, we validated the Random Forest model that learned only the eight features' data at each temperature, and the mean accuracies are shown in the Table 11 . We only showed the representative range $30^{\circ} \mathrm{C}$ to $45^{\circ} \mathrm{C}$, because the results in range $20^{\circ} \mathrm{C}$ to $60^{\circ} \mathrm{C}$ is similar to the results in representative range. The rows of Table 11 shows the training data, and the columns show the testing data in each temperature. For instance, from Table 11, the mean accuracy was $80.5 \%$ when a model constructed using the training data of $30^{\circ} \mathrm{C}$ classified the testing data of $45^{\circ} \mathrm{C}$. Hence, we confirmed that the mean accuracy decreases as the difference between the training data temperature and the testing data temperature increases.

Next, we show the mean accuracies of the learned Random Forest model in case that temperature is added to one of the feature. As shown in Table 12, the model achieved $99 \%$ of accuracy in all data from $30^{\circ} \mathrm{C}$ to $45^{\circ} \mathrm{C}$. Therefore, we conclude that it is possible to construct a robust learned model against feature drift due to drift of temperature by adding temperature as a feature.

\subsection{Detection time evaluation}

In this section, we evaluate the detection time of PLI-TDC from feature extraction phase to classification phase. First, we describe the details of the experimental device. The experiment was conducted on a Raspberry Pi model B+ with $1.2 \mathrm{GHz}$ 64-bit quad-core Cortex-A53 CPU, 1GB RAM, and Debian 10. In our experiment, PLI-TDC executed the inference of the Random Forest classifier (\# of tree is 50) implemented in $\mathrm{C}++$ per receiving the CAN messages. As a result, the time of the feature extraction phase was $13.590 \mu \mathrm{s}$, and the classification phase was $50.217 \mu$ s. Therefore, we concluded that the detection time in PLI-TDC is $63.807 \mu \mathrm{s}$.

\section{DISCUSSION}

\subsection{Identification / detection accuracy}

The mean accuracies of PLI-TDC in CAN bus prototype and realvehicle were $99.67 \%$ and $97.04 \%$, respectively. The results of classifying the data observed with the same time-resolution $(20 \mathrm{~ns})$ as
Divider [22] were $81.43 \%$ and $76.75 \%$, respectively. Besides, we evaluated the mean accuracy rate of PLI-TDC under different timeresolution in the CAN bus prototype. We can change the timeresolution by regarding multiple delay cells in TDC as one delay-cell. For example, when delay-time per one delay-cell is $0.154 \mathrm{~ns}$, we can obtain the time-resolution $0.616 \mathrm{~ns}$ by regarding four delay-cells as one delay-cell. Table 13 shows the mean accuracy of PLI-TDC under different time-resolutions. Also, the eight features selected by Relief-F were used for Random Forest classifier. As shown in Table 13, we confirmed that the mean accuracy is increased by improving the time-resolution. Therefore, we concluded that the mean accuracy of PLI-TDC improves with high time-resolution. Since we confirmed that the mean accuracy is increased by improving the time-resolution, we will try the wave union TDC [27] which has a higher resolution than our TDC as future work.

In addition, we discuss a comparison of accuracy among some voltage-based methods and PLI-TDC with Table 14. The accuracy of PLI-TDC is lower than Scission and EASI. Particularly, EASI can identify the highest accuracy and few numbers of samplings. However, in EASI, it is required to change the sampling rate, because the required sampling rate depends on the length of the payload. On the other hand, PLI-TDC can reliably measure the delay-time without the additional effort, regardless of the data field length. In addition, changes in the voltages may be caused by electromagnetic interference sources. Therefore, voltage-based IDS may cause unexpected false alarms. In the contrast, PLI-TDC can mitigate this problem because the delay-time is a time-domain characteristic not directly influenced by EMI. In addition, manipulation of the delay-time by a remote attacker is expected to be challenging, as the attacker would have to control the timing of the CAN signal with pico-second precision from the software-layer.

Even with a limited number of ECUs in the real-vehicle, the accuracy of PLI-TDC is still $97.04 \%$. The $2.96 \%$ inaccuracy is due to two ECUs with almost the same delay-time (i.e., ECU3 and ECU5 in the real vehicle). As result, PLI-TDC may generate many false alarms per day. While PLI-TDC achieved the lowest B.N.S. and W.N.S. close to the theoretical limit (Table 14), it means that PLITDC has the potential to realize a highly accurate physical-layer IDS with little additional computational complexity in combination with multiple physical characteristics such as voltage and delaytime. Thus, we will try to combine physical-characteristics to solve the problem of false alarms as future work.

\subsection{Number of samplings}

Next, we discuss the number of samplings performed by sender identification methods for each CAN message. Since the number of samplings to be processed per one message influences the performance of PLI such as memory usage and detection time, it is required to reduce the number of samplings in PLI.

The number of samplings per one CAN message for Choi's method, Scission, and SIMPLE depends on the length of the data field. Thus, we consider the case when the data field is the shortest ( 0 byte) and longest ( 8 byte). If the data field is the shortest ( 0 byte), the length of CAN message from SOF to CRC delimiter is 34 bit from Fig. 1. Also, when the bit rate of $\mathrm{CAN}$ is $500 \mathrm{kbps}$, the transmission time for 1 bit is $2 \mu \mathrm{s}$. Hence, the sampling rate of each method is 
Table 11: Mean accuracies under different temperature in CAN bus prototype (eight features).

\begin{tabular}{|c|c|c|c|c|c|c|c|c|c|c|c|c|c|c|c|c|c|}
\hline & \multicolumn{16}{|c|}{ Testing data } \\
\hline & & $30^{\circ} \mathrm{C}$ & $31^{\circ} \mathrm{C}$ & $32^{\circ} \mathrm{C}$ & $33^{\circ} \mathrm{C}$ & $34^{\circ} \mathrm{C}$ & $35^{\circ} \mathrm{C}$ & $36^{\circ} \mathrm{C}$ & $37^{\circ} \mathrm{C}$ & $38^{\circ} \mathrm{C}$ & $39^{\circ} \mathrm{C}$ & $40^{\circ} \mathrm{C}$ & $41^{\circ} \mathrm{C}$ & $42^{\circ} \mathrm{C}$ & $43^{\circ} \mathrm{C}$ & $44^{\circ} \mathrm{C}$ & $45^{\circ} \mathrm{C}$ \\
\hline \multirow{16}{*}{ 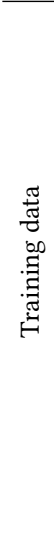 } & $30^{\circ} \mathrm{C}$ & 99.8 & 97.2 & 95.9 & 95.5 & 96.0 & 96.5 & 96.1 & 94.4 & 95.7 & 94.8 & 96.0 & 96.2 & 96.3 & 90.8 & 84.4 & 80.5 \\
\hline & $31^{\circ} \mathrm{C}$ & 96.1 & 99.7 & 95.7 & 95.9 & 95.9 & 96.5 & 96.0 & 94.6 & 95.7 & 95.0 & 96.2 & 96.2 & 96.5 & 91.2 & 85.2 & 82.3 \\
\hline & $32{ }^{\circ} \mathrm{C}$ & 96.4 & 97.3 & 99.5 & 96.4 & 96.3 & 97.1 & 96.8 & 95.6 & 96.4 & 95.9 & 96.5 & 96.4 & 96.6 & 91.2 & 84.2 & 80.2 \\
\hline & $33^{\circ} \mathrm{C}$ & 95.6 & 96.6 & 96.4 & 99.7 & 96.7 & 96.9 & 96.8 & 95.6 & 96.2 & 95.7 & 96.6 & 96.6 & 96.7 & 91.6 & 85.5 & 83.2 \\
\hline & $34^{\circ} \mathrm{C}$ & 95.3 & 96.4 & 95.7 & 96.0 & 99.8 & 97.2 & 97.1 & 95.7 & 96.4 & 95.9 & 96.7 & 96.9 & 97.0 & 92.1 & 86.0 & 83.9 \\
\hline & $35^{\circ} \mathrm{C}$ & 95.7 & 96.7 & 96.2 & 96.3 & 97.3 & 99.9 & 97.6 & 97.1 & 97.4 & 96.8 & 97.4 & 97.4 & 97.4 & 93.0 & 87.5 & 84.4 \\
\hline & $36^{\circ} \mathrm{C}$ & 94.6 & 95.7 & 95.1 & 95.9 & 96.9 & 97.4 & 99.9 & 97.5 & 97.6 & 97.3 & 97.5 & 97.4 & 97.5 & 93.5 & 87.8 & 85.3 \\
\hline & $37^{\circ} \mathrm{C}$ & 93.9 & 94.4 & 94.5 & 94.4 & 95.9 & 97.0 & 97.5 & 99.9 & 98.2 & 98.1 & 98.1 & 98.0 & 97.9 & 94.0 & 89.0 & 86.3 \\
\hline & $38^{\circ} \mathrm{C}$ & 91.9 & 93.9 & 93.6 & 93.0 & 94.9 & 95.9 & 96.6 & 97.8 & 99.8 & 97.9 & 98.1 & 98.1 & 97.9 & 94.1 & 89.4 & 85.7 \\
\hline & $39^{\circ} \mathrm{C}$ & 89.7 & 92.1 & 92.7 & 91.7 & 93.9 & 94.9 & 95.2 & 96.5 & 97.4 & 99.9 & 98.4 & 98.4 & 98.5 & 95.9 & 91.5 & 88.3 \\
\hline & $40^{\circ} \mathrm{C}$ & 89.8 & 92.4 & 92.3 & 91.2 & 92.5 & 93.4 & 94.4 & 95.8 & 97.4 & 98.3 & 99.9 & 98.7 & 98.8 & 96.6 & 93.1 & 89.7 \\
\hline & $41^{\circ} \mathrm{C}$ & 87.2 & 90.3 & 90.9 & 89.3 & 91.5 & 92.9 & 93.2 & 94.3 & 97.0 & 97.8 & 98.6 & 100.0 & 99.0 & 96.8 & 94.0 & 91.0 \\
\hline & $42^{\circ} \mathrm{C}$ & 86.7 & 89.0 & 89.6 & 88.3 & 90.6 & 91.4 & 92.0 & 93.6 & 96.6 & 97.8 & 98.5 & 98.9 & 100.0 & 98.1 & 95.9 & 93.1 \\
\hline & $43^{\circ} \mathrm{C}$ & 85.2 & 88.4 & 88.8 & 87.0 & 89.6 & 90.4 & 89.8 & 90.6 & 94.2 & 96.4 & 97.1 & 98.2 & 98.9 & 99.9 & 97.3 & 96.3 \\
\hline & $44^{\circ} \mathrm{C}$ & 83.5 & 87.3 & 88.3 & 86.3 & 88.9 & 89.4 & 89.0 & 89.8 & 93.6 & 95.6 & 96.7 & 97.9 & 98.6 & 98.5 & 99.9 & 97.0 \\
\hline & $45^{\circ} \mathrm{C}$ & 83.9 & 87.4 & 88.3 & 86.2 & 88.9 & 89.8 & 89.1 & 89.8 & 93.2 & 95.5 & 96.2 & 97.4 & 98.2 & 97.9 & 97.4 & 99.8 \\
\hline
\end{tabular}

Table 12: Mean accuracies under different temperature in CAN bus prototype (eight features and temperature).

\begin{tabular}{|c|c|c|c|c|c|c|c|c|c|c|c|c|c|c|c|c|}
\hline & \multicolumn{16}{|c|}{ Testing data } \\
\hline & $30^{\circ} \mathrm{C}$ & $31^{\circ} \mathrm{C}$ & $32^{\circ} \mathrm{C}$ & $33^{\circ} \mathrm{C}$ & $34^{\circ} \mathrm{C}$ & $35^{\circ} \mathrm{C}$ & $36^{\circ} \mathrm{C}$ & $37^{\circ} \mathrm{C}$ & $38^{\circ} \mathrm{C}$ & $39^{\circ} \mathrm{C}$ & $40^{\circ} \mathrm{C}$ & $41^{\circ} \mathrm{C}$ & $42^{\circ} \mathrm{C}$ & $43^{\circ} \mathrm{C}$ & $44^{\circ} \mathrm{C}$ & $45^{\circ} \mathrm{C}$ \\
\hline Roboust Model & 99.8 & 99.8 & 99.8 & 99.8 & 99.8 & 99.8 & 99.8 & 99.7 & 99.3 & 99.3 & 99.3 & 99.2 & 99.2 & 99.2 & 99.3 & 99.3 \\
\hline
\end{tabular}

Table 13: Mean accuracy under different time-resolution.

\begin{tabular}{c|cccccc}
\hline $\begin{array}{c}\text { time-resolution } \\
\text { [ns] }\end{array}$ & 20.000 & 7.700 & 3.850 & 1.540 & 0.616 & 0.154 \\
$\begin{array}{c}\text { Mean accuracy } \\
{[\%]}\end{array}$ & 87.20 & 93.02 & 97.47 & 98.31 & 98.46 & 99.67 \\
\hline
\end{tabular}

Table 14: Comparison among voltage-domain based methods in Accuracy of Identification (A.I.), Sampling Rate (S.R.), Best Number of Samplings per message (B.N.S.), Worst Number of Samplings per message (W.N.S.), Time Complexity in Feature extraction (T.C.F.), Detection Time (D.T.).

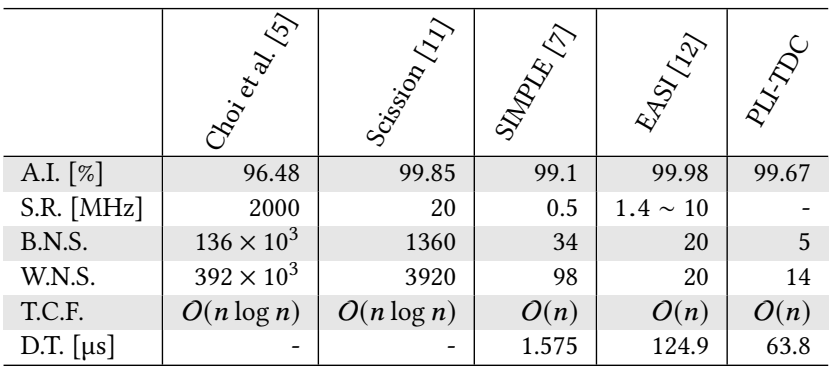

multiplied by $34 \times 2 \times 10^{-6}$. Thus, for instance, the best number of samplings in Choi's method is $2000 \mathrm{MHz} \times 34 \times 2 \times 10^{-6}=136 \times 10^{3}$. As a result, the best number of samplings per one CAN message in Choi's method, Scission, and SIMPLE is $136 \times 10^{3}, 1360$, and 34 , respectively. Similarly, if the data field is the longest ( 8 byte), the length of the CAN message from SOF to CRC delimiter is 98 bit.
Therefore, the worst number of samplings is $392 \times 10^{3}, 3920$, 98 respectively. Also, EASI's number of samplings is 20 for both best and worst. The number of samplings per message in PLI-TDC depends on the number of signal transitions from 0 to 1 , not the length of the data field. Consequently, The minimum and the maximum number of samplings of PLI-TDC are discussed with Arbitration ID 0x000, which has a small number of bit transitions, and Arbitration ID 0x555, which has a large number of transitions. As a result, the best number of samplings reached 5 . The worst number of samplings reached 14. The results show that PLI-TDC has the least number of samplings at the data acquisition phase; in other words, PLI-TDC has the smallest $n$ at the feature extraction phase. Hence, the feature extraction of PLI-TDC is possible with light processing.

Finally, we discuss computational complexity. The method of Choi et al. and Scission use time and frequency domain features. Therefore, these methods need $O(n \log n)$ time because these methods perform Fourier Transforms to calculate the frequency domain feature. Also, the feature extraction phases of SIMPLE and EASI takes $O(n)$. Because PLI-TDC uses statistic features in Table 2, PLITDC needs $O(n)$. Therefore, we confirmed that the computational complexities of SIMPLE, EASI, and PLI-TDC are lower than the computational complexities of other methods.

From these comparisons among related works, we confirmed that PLI-TDC can reduce the amount of data in the data acquisition phase than the other voltage-based methods.

\subsection{Detection time}

We discuss whether PLI-TDC can identify all messages without spilling messages. In Sec. 5.5, the evaluation of detection time was conducted on a Raspberry Pi model B + which used runs at a $1.2 \mathrm{GHz}$ 
64-bit quad-core Cortex-A53 CPU. This environment is the same as a specification of the system used for autonomous, in-vehicle infotainment, and gateway function [23]. Thus, the experiment environment of PLI-TDC can be implemented in actual vehicles.

As described in Sec. 5.5, PLI-TDC's detection time is $63.807 \mu \mathrm{s}$. A minimum interval of between two CAN frames is $222 \mu \mathrm{s}$ in CAN of $500 \mathrm{kbps}$. Since the detection time is lower than the minimum interval in CAN, we conclude that PLI-TDC can validate all CAN messages without spilling messages.

\subsection{Stability and life-cycle}

In this section, we describe the stability of the delay-time and lifecycle of the learned model in PLI-TDC. PLI-TDC may not adapt long-term gradual feature drift caused by material wear and so on. Thus, we collected the $24 \mathrm{~GB}$ of data for 86 days from the realvehicle. However, we could not find any gradual drift of delay-time in the 86 days. Hence, we confirmed that PLI-TDC has the stability of feature at least 86 days. We also recommend updating a delaytime based model after 86 days from deploying the model. Besides, as the other feature drift, in case of sudden drift caused by some fail, we can update immediately a learned model because Random Forest classifiers can learn data fastly with a few seconds [29].

\section{CONCLUSION}

To avoid the security risk on automobiles, PLIs in CAN have been proposed. To meet requirements of high accuracy, the small number of samplings of feature, and robustness against temperature change, we proposed a delay-time based sender identification called PLITDC which is higher accuracy than Divider and gets the features with a few sampling. In addition, PLI-TDC realizes temperaturerobustness by learning the temperature as one of the features. We implemented the experimental devices using FPGA and microcomputer to verify our method for identification. As a result, we confirm that PLI-TDC achieved a mean accuracy of $99.67 \%$ in the CAN bus prototype and of $97.04 \%$ in the real-vehicle. We have released our research [21] in the hope to promote research on sender identification. In our future work, we will try PLI-TDC using the wave union TDC and combine physical-characteristics.

\section{REFERENCES}

[1] ams. 2020. AS6500 Time-to-Digital Converter. https://ams.com/ja/as6500. (Accessed on 10/25/2020)

[2] Yasuo Arai and Masahiro Ikeno. 1996. A Time Digitizer CMOS Gate-Array with a 250 ps Time Resolution. IEEE fournal of Solid-State Circuits 31, 2 (1996), 212-220.

[3] Kyong-Tak Cho and Kang G Shin. 2016. Fingerprinting Electronic Control Units for Vehicle Intrusion Detection. In 25th USENIX Security Symposium (USENIX Security 16). 911-927.

[4] Kyong-Tak Cho and Kang G Shin. 2017. Viden: Attacker Identification on InVehicle Networks. In Proceedings of the 2017 ACM SIGSAC Conference on Computer and Communications Security. ACM, 1109-1123.

[5] Wonsuk Choi, Hyo Jin Jo, Samuel Woo, Ji Young Chun, Jooyoung Park, and Dong Hoon Lee. 2018. Identifying ECUs Using Inimitable Characteristics of Signals in Controller Area Networks. IEEE Transactions on Vehicular Technology 67, 6 (2018), 4757-4770.

[6] The MITRE Corporation. 2020. CVE-2020-5551. https://cve.mitre.org/cgi-bin/ cvename.cgi?name=CVE-2020-5551. (Accessed on 04/26/2020).

[7] Mahsa Foruhandeh, Yanmao Man, Ryan Gerdes, Ming Li, and Thidapat Chantem 2019. SIMPLE: Single-Frame Based Physical Layer Identification for Intrusion Detection and Prevention on In-Vehicle Networks. In Proceedings of the 35th Annual Computer Security Applications Conference. 229-244.

[8] Robert Bosch GmbH. 1991. CAN Specification Version 2.0. http://esd.cs.ucr.edu/ webres/can20.pdf. (Accessed on 07/08/2019).
[9] Anthony Van Herrewege, Dave Singelee, and Ingrid Verbauwhede. 2011. CANAuth-A Simple, Backward Compatible Broadcast Authentication Protocol for CAN Bus. In ECRYPT Workshop on Lightweight Cryptography, Vol. 2011. 1-7.

[10] Min-Joo Kang and Je-Won Kang. 2016. Intrusion Detection System Using Deep Neural Network for In-Vehicle Network Security. PloS one 11, 6 (2016), e0155781.

[11] Marcel Kneib and Christopher Huth. 2018. Scission: Signal Characteristic-Based Sender Identification and Intrusion Detection in Automotive Networks. In Proceedings of the 2018 ACM SIGSAC Conference on Computer and Communications Security. ACM, 787-800.

[12] Marcel Kneib, Oleg Schell, and Christopher Huth. 2020. EASI: Edge-Based Sender Identification on Resource-Constrained Platforms for Automotive Networks. In Proceedings Network and Distributed System Security Symposium. 1-16.

[13] Igor Kononenko. 1994. Estimating Attributes: Analysis and Extensions of RELIEF. In European conference on machine learning. Springer, 171-182.

[14] Mirco Marchetti and Dario Stabili. 2017. Anomaly Detection of CAN Bus Messages through Analysis of ID Sequences. In 2017 IEEE Intelligent Vehicles Symposium (IV). IEEE, 1577-1583.

[15] Mirco Marchetti, Dario Stabili, Alessandro Guido, and Michele Colajanni. 2016. Evaluation of Anomaly Detection for In-Vehicle Networks through InformationTheoretic Algorithms. In 2016 IEEE 2nd International Forum on Research and Technologies for Society and Industry Leveraging a better tomorrow (RTSI). IEEE, $1-6$.

[16] Charlie Miller and Chris Valasek. 2015. Remote Exploitation of An Unaltered Passenger Vehicle. Black Hat USA 2015 (2015), 1-91.

[17] Pal-Stefan Murvay and Bogdan Groza. 2014. Source Identification Using Signal Characteristics in Controller Area Networks. IEEE Signal Processing Letters 21, 4 (2014), 395-399.

[18] Pal-Stefan Murvay and Bogdan Groza. 2020. TIDAL-CAN: Differential Timing Based Intrusion Detection and Localization for Controller Area Network. IEEE Access 8 (2020), 68895-68912.

[19] Sen Nie, Ling Liu, and Yuefeng Du. 2017. Free-Fall: Hacking Tesla from Wireless to CAN Bus. Black Hat USA 2017 (2017), 1-16.

[20] Stefan Nürnberger and Christian Rossow. 2016. -vatiCAN-Vetted, Authenticated CAN Bus. In International Conference on Cryptographic Hardware and Embedded Systems. Springer, 106-124.

[21] Shuji Ohira. 2020. PLI-TDC. https://github.com/shuji-oh/PLI_TDC_for_CAN. (Accessed: 2020-10-25).

[22] Shuji Ohira, Araya Kibrom Desta, Tomoya Kitagawa, Ismail Arai, and Kazutoshi Fujikawa. 2020. Divider: Delay-Time Based Sender Identification in Automotive Networks. In IEEE 44th Annual Computer Software and Applications Conference (COMPSAC). IEEE, 1490-1497.

[23] James Scobie. 2018. A Starter's Guide to Arm Processing Power in Automotive - Embedded blog - System - Arm Community. https://community.arm.com/developer/ip-products/system/b/embeddedblog/posts/a-starters-guide-to-arm-processing-power-in-automotive. (Accessed on $05 / 31 / 2020$ ).

[24] Hyun Min Song, Ha Rang Kim, and Huy Kang Kim. 2016. Intrusion Detection System Based on the Analysis of Time Intervals of CAN Messages for In-Vehicle Network. In 2016 international conference on information networking (ICOIN). IEEE, 63-68.

[25] Jian Song, Qi An, and Shubin Liu. 2006. A High-Resolution Time-to-Digital Converter Implemented in Field-Programmable-Gate-Arrays. IEEE Transactions on Nuclear Science 53, 1 (2006), 236-241.

[26] Adrian Taylor, Sylvain Leblanc, and Nathalie Japkowicz. 2016. Anomaly Detection in Automobile Control Network Data with Long Short-Term Memory Networks. In 2016 IEEE International Conference on Data Science and Advanced Analytics (DSAA). IEEE, 130-139.

[27] Jinyuan Wu and Zonghan Shi. 2008. The 10-ps Wave Union TDC: Improving FPGA TDC Resolution beyond Its Cell Delay. In 2008 IEEE Nuclear Science Symposium Conference Record. IEEE, 3440-3446.

[28] Wufei Wu, Yizhi Huang, Ryo Kurachi, Gang Zeng, Guoqi Xie, Renfa Li, and Keqin Li. 2018. Sliding Window Optimized Information Entropy Analysis Method for Intrusion Detection on In-Vehicle Networks. IEEE Access 6 (2018), 45233-45245.

[29] Mahmut Yazici, Shadi Basurra, and Mohamed Gaber. 2018. Edge Machine Learning: Enabling Smart Internet of Things Applications. Big Data and Cognitive Computing 2, 3 (2018), 1-17.

[30] Jia Zhou, Prachi Joshi, Haibo Zeng, and Renfa Li. 2019. BTMonitor: Bit-TimeBased Intrusion Detection and Attacker Identification in Controller Area Network. ACM Transactions on Embedded Computing Systems (TECS) 18, 6 (2019), 1-23. 